\title{
Developing a cells, tissue and organ surveillance system
}

\author{
Hong $\mathrm{Z}^{1 *}$, Kenny $\mathrm{P}^{1}$, Hyson $\mathrm{C}^{1}$, Archibald $\mathrm{C}^{1}$ \\ ${ }^{1}$ Centre for Communicable Diseases and Infection Control, Public Health Agency of Canada, Ottawa, ON \\ * Correspondence: zhiyong.hong@phac-aspc.gc.ca
}

\section{Abstract}

Background: An increasing number of cell, tissue and organ transplant procedures take place each year in Canada, including procedures in clinics, physician and dental offices. The Public Health Agency of Canada (the Agency) is leading the development of a Cell, Tissue and Organ Surveillance System (CTOSS).

Objective: To create timely, useful and relevant national-level transplantation adverse event data by supporting the development and / or enhancement of provincial and territorial data collection systems.

Methods: Minimum data elements and definitions were established for tissues based on definitions established in the European Union and the United States. Data collection on adverse events related to human allograft tissue transplants began in April 2011 at pilot sites in Alberta, Ontario, Quebec, New Brunswick and Nova Scotia.

Results: By December 2013, eight tissue transplantation adverse events were reported. Seven involved corneal tissue and one involved cardiovascular tissue.

Conclusion: A fully developed CTOSS could increase Canadian capacity to improve patient safety. Data collection and analysis could increase the potential for a better understanding of transplantation adverse events, subsequently inform the development of strategies for overall prevention and reduce the severity of such events. The next steps in developing CTOSS will be to establish data elements and definitions for the cell and organ transplant components of the system and increase the number of pilot sites.

\section{Introduction}

An increasing number of cell, tissue and organ transplant procedures take place each year in Canada, including procedures in clinics, physician and dental offices (1). Over 90,000 tissue allografts are distributed for transplantation, including musculoskeletal, vascular, skin, cardiac and corneal tissues (2). Canadian Blood Services predicts that the need for organ transplants will increase significantly over the next two decades (3). The Public Health Agency of Canada (the Agency) is leading the development of a Cell, Tissue and Organ Surveillance System (CTOSS) that will aim to improve transplantation safety for Canadians by capturing and analyzing adverse event data and disseminating the resulting knowledge. Transplantation adverse event surveillance data is critical to our ability to improve patient safety through the development of appropriate programs and policies. Once fully developed, the system will collect data on moderate and severe adverse events in order to monitor trends in known and emerging risks and reduce the transmission of infectious diseases due to transplantation. The objective of this article is to summarize progress toward the development of the three component transplantation adverse event surveillance systems (4).

\section{Methods}

Minimum data elements and definitions of serious adverse events were developed in conjunction with definitions established in the European Union and the United States. For grading of severity related to the adverse event, the spectrum of nil, non-serious, serious, life-threatening and death were defined and applied to the case report. The grading system for imputability was applied to assess the probability that an adverse event in a recipient may be attributed to the process of donation or clinical application of the tissue applied. Data collection on adverse events 
related to human allograft tissue transplants began in April 2011 at pilot sites in Alberta, Ontario, Quebec, New Brunswick and Nova Scotia. The mechanisms used to gather transplantation adverse event reports vary among the participating pilot sites.

\section{Results}

By December 2013, eight tissue transplantation adverse events were reported and the majority involved corneal tissue (Table 1). There were five corneal tissue transplantation adverse events (AEs) reported to the Agency from the Alberta pilot site since April 1 of 2011 and one corneal tissue-related adverse event was reported to the Agency from the Nova Scotia pilot site in 2011. One cardiovascular tissue-related serious adverse event was reported to the Agency from the Quebec pilot site in 2012 as well as a corneal tissue-related adverse event in 2013. Overall, most surveillance sites delivered complete transplantation AE reports and annual reports. It generally took three to six months for the site and tissue establishment to conduct a complete investigation of a suspected transplantation AE. There were several factors which affected the reporting of transplantation AEs including the expertise and experience of the treating physician in the detection of transplantation AEs in recipients, the effectiveness of coordination between procurement organizations and the hospitals as well as the workload of the coordinators.

Table 1: Overview of tissue transplantation adverse events by CTOSS ${ }^{1}$ sites from April 2011 to December 2013

\begin{tabular}{|l|c|c|c|c|c|}
\hline Year & Site 1 & Site 2 & Site 3 & Site 4 & Site 5 \\
\hline $\mathbf{2 0 1 1}$ & 0 & 0 & 0 & 0 & 1 \\
\hline $\mathbf{2 0 1 2}$ & 4 & 0 & 1 & 0 & 0 \\
\hline $\mathbf{2 0 1 3}$ & 1 & 0 & 1 & 0 & 0 \\
\hline Total & $\mathbf{5}$ & $\mathbf{0}$ & $\mathbf{2}$ & $\mathbf{0}$ & $\mathbf{1}$ \\
\hline
\end{tabular}

${ }^{1}$ CTOSS $=$ Cell, Tissue and Organ Surveillance System

\section{Conclusion}

Canada's Cell, Tissue and Organ Surveillance System increases our capacity to identify common adverse events associated with transplantation. Improved data collection and analysis will increase our understanding of transplantation adverse events and inform the development of strategies to prevent or minimize these events. The next step in developing CTOSS will be to establish data elements and definitions for the cell and organ transplant components of the system and expand the number of sites participating in CTOSS.

\section{Acknowledgements}

The Agency would like to thank the following healthcare establishments for their contributions toward the surveillance of tissue- and organ-related adverse events: Alberta Health's Transplant Services, Héma-Québec and the Ministère de la Santé et des Service sociaux du Québec, Sunnybrook Hospital in Toronto Ontario, the New Brunswick Eye and Tissue Bank and the Nova Scotia Provincial Blood Coordinating Program. The Agency also greatly appreciated the comments and suggestions by the Marketed Health Products Directorate of the Health Products and Food Branch at Health Canada and Canadian Blood Services during the process of preparing this document. 


\section{Conflict of interest}

None

\section{Funding}

Funding was provided by the Public Health Agency of Canada.

\section{References}

(1) Canadian Organ Replacement Register, 2013. Toronto: Canadian Institute for Health Information; 2013.

(2) Canadian Council for Donation and Transplantation. Surveillance of recipients of organ and tissue transplants; February 2008.

(3) Canadian Blood Services, Call to action - A Strategic plan to improve organ and tissue donation and transplantation performance for Canadians. Toronto: Canadian Blood Services; 2011.

(4) Public Health Agency of Canada. Cells, Tissue and Organ Surveillance System: Project progress report April 2007December 2013. Ottawa: Centre for Communicable Disease and Infection Control; 2013. http://publications.gc.ca/collections/collection_2014/aspc-phac/HP40-120-2014-eng.pdf. 